\section{IMPACTO DE "EL NIÑO 1997-98" SOBRE LAS COMUNIDADES VEGETALES DE LAS LOMAS DEL CERRO CAMPANA, TRUJILLO - PERÚ}

\author{
IMPACT OF "EL NIÑO 1997-98" ON LOMAS VEGETAL COMMUNITIES OF CERRO \\ CAMPANA, TRUJILLO - PERU
}

Gustavo Ywanaga y Carlos Bocanegra*

\begin{abstract}
Periodical fieldwork were carried out to Lomas of Cerro Campana at $7^{\circ} 58^{\prime} 57^{\prime \prime} \mathrm{LS}$ and $79^{\circ} 06^{\prime} 15^{\prime \prime} \mathrm{LW}$, Trujillo, La Libertad, Peru, between October 1997 and August 1998, in order to analyze vegetal cover.

The vegetation was dominated by Tiquilia dichotoma, Calandrinia ciliata and Exodeconus prostatus, during "El Niño 1997-98", overcame to the dominant species of non-Niño year, hepatics, líchens, cactaceous and bromeliaceous.
\end{abstract}

Las lomas costeras constituyen formaciones vegetales particulares, que se distribuyen a lo largo de la costa sudamericana del Pacífico, entre los 8 y $30^{\circ}$ de latitud sur. El Cerro Campana es particularmente interesante para investigar el impacto del evento "El Niño 1997-98", por constituir el límite norte de dicho tipo de formación vegetal.

Las lomas del Cerro Campana a $7^{\circ} 58^{\prime} 57^{\prime \prime}$ LS y $79^{\circ} 06^{\prime} 15^{\prime \prime} \mathrm{LW}$, ubicadas en la Provincia de Trujillo, Departamento de La Libertad, Perú, fueron visitadas periódicamente de octubre de 1997 a agosto de 1998. El estudio consistió en reconocimiento general de las lomas, análisis de cobertura vegetal empleando el método del cuadrado, registro fotográfico y colecta de muestras vegetales. La información meteorológica fue

* Universidad Nacional de Trujillo proporcionada por la Estación Trujillo del proyecto Globos Piloto de la NOAA y la identificación del material biológico colectado se hizo con ayuda del personal del Herbarium Truxillense de la Universidad Nacional de La Libertad.

Durante el evento "El Niño 1997-98" se colectaron un total de 32 especies vegetales (Tab. 1), de las cuales 3 especies fueron claramente dominantes: Tiquilia dichotoma (26\%) y Calandrinia ciliata (15\%), ambas presentes todo el año, pero no dominantes en condiciones normales; y Exodeconus prostatus ( $30 \%$ ), que sólo se encuentra durante el otoño en años normales, en esta oportunidad estuvo activa más de 12 meses, bajo las condiciones anómalas de humedad y temperatura. Las bromeliáceas, cactáceas, hepáticas y otros mostraron valores de dominancia bajos. Este panorama contrasta 
Tabla 1. Especies vegetales colectadas durante la ocurrencia de "El Niño 1997-98".

\begin{tabular}{|c|c|c|}
\hline Familias & Especies & Hábito \\
\hline \multirow[t]{6}{*}{ Asteraceae } & Ageratina azangaroensis & arbusto \\
\hline & Flaveria bidentis & hierba \\
\hline & Flaveria sp. & hierba \\
\hline & Senecio multifidum & hierba \\
\hline & Sonchus oleraceus & hierba \\
\hline & Verbesina saubinetioides & arbusto \\
\hline \multirow[t]{2}{*}{ Boraginaceae } & Heliotropium angiospermum & hierba \\
\hline & Tiquilia dichotoma & hierba \\
\hline \multirow[t]{2}{*}{ Bromeliaceae } & Tillandsia latifolia & hierba \\
\hline & Tillandsia purpurea & hierba \\
\hline \multirow[t]{3}{*}{ Cactaceae } & Haageocereus lanugispinus & cactus \\
\hline & Melocactus peruvianus & cactus \\
\hline & Neoraimondia arequipensis & cactus \\
\hline Capparaceae & Capparis scabrida & hierba \\
\hline \multirow[t]{2}{*}{ Chenopodiaceae } & Atriplex rotundifolia & hierba \\
\hline & Chenopodium ambrosioides & hierba \\
\hline Cucurbitaceae & Cucumis dipsaceus & hierba \\
\hline Lythraceae & Lythrum maritimum & hierba \\
\hline Malvaceae & Urocarpidium peruvianum & hierba \\
\hline Nyctaginaceae & Allionia incarnata & hierba \\
\hline \multirow[t]{2}{*}{ Poaceae } & Aristida adscensionis & hierba \\
\hline & Phragmites australis & hierba \\
\hline \multirow[t]{2}{*}{ Portulacaceae } & Calandrinia ciliata & hierba \\
\hline & Cistanthe ruizii & hierba \\
\hline \multirow[t]{6}{*}{ Solanaceae } & Datura stramonium & hierba \\
\hline & Exodeconus prostratus & hierba \\
\hline & Grabowskia boerhaaviaefolia & arbusto \\
\hline & Leptoglossis schwenkioides & hierba \\
\hline & Nicandra physalodes & hierba \\
\hline & Nolana humifusa & hierba \\
\hline Tiliaceae & Corchorus hirtus & hierba \\
\hline Urticaceae & Parietaria debilis & hierba \\
\hline
\end{tabular}

drásticamente con la vegetación dominante reportada para años de no-Niño, en los que dominan las hepáticas, líquenes, cactáceas y bromeliáceas, según Sagástegui et al. (1998).

\section{LITERATURA CITADA}

Sagástegui, A.: J. Mostaceros y S. López. 1988. Fitoccología del Cemo Campana. Bol. Srat Bot. La Libertad $14(1): 1-47$. 